\title{
Hipertensão arterial e trabalho: fatores de risco
}

\author{
Hypertension and work: risk factors
}

\author{
Roberta Coimbra Velez de Andrade', Rita de Cássia Pereira Fernandes²
}

\begin{abstract}
RESUMO | Contexto: A hipertensão arterial sistêmica (HAS) constitui um dos principais problemas de saúde pública da atualidade. Sabe-se que a etiologia da HAS é multifatorial. Com relação aos fatores associados ao desenvolvimento dessa patologia, tem-se discutido na literatura a exposição a fatores ocupacionais como um risco independente para o desenvolvimento de hipertensão. Objetivos: Este estudo teve por objetivo realizar uma revisão de literatura sobre fatores ocupacionais e hipertensão arterial sistêmica. Métodos: Uma consulta de artigos selecionados publicados num período de cinco anos (2008-2012) nas principais bases de dados eletrônicas: SciELO, MEDLINE e LILACS. Resultados: Dentre os fatores ocupacionais analisados, o ruído se destacou como possivelmente associado à hipertensão arterial, seguido de trabalho em turnos. Conclusão: Apesar de os resultados apontarem ruído e trabalho em turnos como fatores possivelmente associados à hipertensão arterial, são necessários mais estudos sobre o tema HAS e fatores ocupacionais — para ampliar a compreensão dessa problemática.
\end{abstract}

Palavras-chave I hipertensão; saúde do trabalhador; fatores de risco.

ABSTRACT I Context: Systemic arterial hypertension (SAH) is one of the main public health issues in our time. The etiology of SAH is known to be multifactorial. Regarding the factors associated with the development of this condition, literature has discussed the exposure to occupational factors as an independent risk for the development of hypertension. Objectives: This study aimed at conducting a literature review about occupational factors and systemic arterial hypertension. Methods: A search for papers published in a five-year period (2008-2012) was carried out in the main electronic databases: SciELO, MEDLINE, and LILACS. Results: Among the occupational factors analyzed, noise stood out as being possibly associated with arterial hypertension, followed by working shifts. Conclusion: Even though the results indicate noise and working shifts as factors that are possibly associated with arterial hypertension, further studies about the subject are required - SAH and occupational factors - to improve the understanding of this theme. Keywords I hypertension; occupational health; risk factors. 


\section{INTRODUÇÃO}

A hipertensão arterial sistêmica (HAS) constitui um importante problema de saúde pública da atualidade, com uma prevalência de $22,7 \%$ (em 2011) para a população brasileira com idade superior a 18 anos $^{1}$.É o principal fator de risco para doenças cardiovasculares ${ }^{2}$ e foi a primeira causa de morte no Brasil no ano de 2008, segundo o Ministério da Saúde 3 . De acordo com as VI Diretrizes Brasileiras de Hipertensão ${ }^{4}$, a HAS pode ser definida como o aumento sustentado da pressão arterial (PA) sistólica ou diastólica com medida igual ou maior a 140 e $90 \mathrm{mmHg}$, respectivamente, detectado em duas aferições realizadas em momentos distintos.

Sabe-se que a etiologia da HAS émultifatorial. Entre os fatores associados ao seu desenvolvimento estão idade, gênero, etnia, excesso de peso e obesidade, ingestão de sal e de álcool, sedentarismo, hereditariedade e fatores socioeconômicos ${ }^{4}$. Ademais, discute-se na literatura internacional a exposição a fatores ocupacionais como um risco independente para o desenvolvimento de hipertensão ${ }^{5}{ }^{27}$. Alguns estudos apontam ruído ${ }^{5 \cdot 12}$, trabalho em turnos ${ }^{5,11,13-17}$ exposição a alguns agentes químicos ${ }^{8-11}$ como associados ao aumento dos níveis pressóricos. Outros artigos relacionam o estresse no ambiente de trabalho ${ }^{13,18-22}$ como um fator biopsicossocial associado à hipertensão. Nesse contexto, éintroduzido o conceito biológico do estresse e seu efeito sistêmico, no qual a ativação adrenérgica, por meio da liberação de hormônios reguladores da pressão arterial, promoveria vasoconstricção periférica e, consequentemente, elevação dos níveis pressóricos ${ }^{19-21}$.

Entretanto, há controvérsias sobre o assunto e a devida relevância dos fatores de riscos ocupacionais na abordagem da hipertensão pode estar subestimada. Essa premissa é válida em face da importância do trabalho no cotidiano do indivíduo, que tem parte significativa da sua vida dedicada à atividade laboral e a partir dela estrutura suas relações socioeconômicas, culturais e, muitas vezes, de estilo de vida.

Diante do exposto, este trabalho foi elaborado com o intuito de realizar uma revisão de literatura sobre exposição a fatores de risco ocupacionais e HAS, com base em publicações num período de cinco anos sobre o tema.

\section{MÉTODOS}

Trata-se de uma revisão de literatura, cujo objetivo foi identificar artigos sobre HAS e possíveis fatores de risco ocupacionais publicados em um período de cinco anos (2008-2012). Foram utilizadas as seguintes bases de dados eletrônicas: The Scientific Electronic Library Online (SciELO), MEDLINE e Literatura Latino-Americana e do Caribe em Ciências da Saúde (LILACS). Para isso, foram efetuadas buscas nessas fontes, usando combinações das seguintes palavras: "hipertensão", "pressão arterial”, “trabalho”, "trabalhador”, “ocupacional”, "ocupação”, "doenças cardiovasculares”, "hypertension”, "bloodpressure", "job", "work", "workers" e "occupational".

Os critérios de inclusão utilizados neste estudo foram: artigos originais; estudos nos quais a pressão arterial foi aferida ou considerada positiva quando havia referência ao uso de anti-hipertensivo; artigos que apresentassem resultados analíticos, com as medidas de associação entre hipertensão e possíveis fatores de risco; e estudos publicados em português, inglês e espanhol. Foram excluídos deste trabalho estudos cujo diagnóstico de hipertensão foi apenas referido, estudos sobre hipertensão arterial que não descreviam os fatores ocupacionais associados à hipertensão e artigos de revisão. Estes últimos, apesar de estarem entre os critérios de exclusão, também foram submetidos à leitura como embasamento para o presente estudo.

Diante dos resultados encontrados, no total 1.794 publicações, realizou-se uma seleção com base inicialmente nos títulos daqueles artigos originais de interesse sobre a temática (relação entre exposição a fatores de risco ocupacionais e hipertensão arterial sistêmica). As 465 publicações retidas nesse processo foram submetidas à verificação de duplicidade e leitura dos seus resumos, com posterior exclusão de 418 artigos por serem estudos que não analisaram a referida associação ou por estarem duplicados.

Com base no descrito, 47 estudos foram inicialmente submetidos à leitura completa e, posteriormente, à avaliação crítica de validade/qualidade científica para definir quais deles seriam de fato incluídos nesta revisão, considerando os critérios de inclusão. Assim, obteve-se um número final de 23 artigos para elaboração deste estudo.

\section{RESULTADOS}

As informações acerca dos estudos já apresentados foram descritas na Tabela 1, agrupadas a partir das seguintes características: título do artigo, desenho do estudo, população estudada, exposição ocupacional analisada e principais achados. 
Tabela 1. Síntese dos estudos sobre fatores ocupacionais associados à hipertensão arterial.

\begin{tabular}{|l|c|c|c|}
\hline Título do artigo & $\begin{array}{c}\text { Desenho } \\
\text { do estudo }\end{array}$ & $\begin{array}{c}\text { n do estudo } \\
\text { População estudada }\end{array}$ & $\begin{array}{c}\text { Tipo de } \\
\text { exposição } \\
\text { ocupacional }\end{array}$ \\
\hline
\end{tabular}

Effect of exposure to occupational noise and shift working on blood pressure in rubber manufacturing company workers ${ }^{5}$
331 trabalhadores de empresa de fabrica-

Estudo transversal ção de borracha em no Irã) em 2010 Yazd (província central

Trabalho em turnos e ruído
Resultados

As maiores taxas de hipertensão e de pressão arterial sistólica e diastólica média foram observadas entre os trabalhadores de turno que foram expostos a ruído superior ao limite permitido. Também houve uma relação significativa entre a exposição simultânea ao ruído superior ao limite permitido e turno de trabalho com hipertensão.

A intensificação do estresse no local de traba-

416 mulheres de quatro grupos de profissionais: agricultura;

Exposing women to workplace stress fac tors as a risk factor for developing arterial
Estudo transversal balconistas; costureiras e representantes médicos, no período de agosto a setembro de 2008, na região de

Lublin, Polônia
Iho teve um impacto considerável sobre o valor da pressão arterial entre o grupo de mulheres representantes médicos do sexo feminino, Estresse bem como entre o grupo de trabalhadores de escritório. Nenhuma associação significativa foi encontrada entre variáveis sociodemográficas e nível geral de exposição ao estresse entre grupos profissionais examinados.
790 trabalhadores da linha de produção em ing loss, occupational noise exposure and hypertensio: a cross-sectional study in male workers ${ }^{6}$
Estudo uma empresa de fabritransversal cação de aviões com 1.094 colaboradores no final de 1998, Taiwan
Ruído

Os resultados sugerem que a perda auditiva de alta frequência é um bom biomarcador de exposição ocupacional a ruído e que a perda auditiva induzida por ruído pode estar associada com o risco de hipertensão.
Noise frequency components and the prevalence of hypertension in workers $^{7}$
Estudo transversal uma fábrica de parafusos em Taichung Taiwan, 2009
Ruído

Ruido

. 
Tabela 1. Continuação.

\begin{tabular}{|l|c|c|c|c} 
Título do artigo & $\begin{array}{c}\text { Desenho } \\
\text { do estudo }\end{array}$ & $\begin{array}{c}\text { n do estudo } \\
\text { População estudada }\end{array}$ & $\begin{array}{c}\text { Tipo de } \\
\text { exposição } \\
\text { ocupacional }\end{array}$ & Resultados \\
\hline
\end{tabular}

Prevalência de hipertensão arterial em militares jovens e fatores associados ${ }^{23}$

Estudo transversal

Blood pressure and working conditions in hospital nurses and nursing assistants. The ORSOSA study ${ }^{13}$
380 militares do sexo masculino entre 19 e 35 anos de idade em uma unidade da Força Aérea Brasileira em São Paulo (SP), entre 2000 e 2001

214 unidades de trabalho com um total de 2.307 enfermeiros e 1.530 auxiliares de Coorte enfermagem de 7 hospitais universitá-rios voluntários franceses em um intervalo de 2 anos (2006 e 2008)
Hierarquia, As variáveis "escolaridade", "hierarquia militar", tempo de "tempo de trabalho" e "renda" não se associaram trabalho ao desfecho.

\section{Exposure to} occupational noise and cardiovascular disease in the United States: the National Health and Nutrition Examination Survey $1999-2004^{9}$
6.307 participantes do Exame Nacional de Saúde e Nutrição

Estudo transversal 1999-2004, com idade $\geq 20$ anos e empregados no momento da entrevista

A diferença entre a pressão arterial sistólica entre os trabalhadores dos turnos diurno e noturno foi $2,5 \mathrm{mmHg}(\mathrm{p}<0,001)$. O questionáEstresse e rio Nursing Work Index-Extended Organization trabalho em (NWI-EO) indicou que a PAS e PAD foi mais turnos fortemente associada a uma relação pobre da equipe $(p<0,01)$. Para uma diferença de um ponto no escore de estresse NWI-EO, a PAS foi maior, em média, 0,2 mmHg.

Comparados com os participantes nunca expostos, os indivíduos cronicamente expostos ao ruído ocupacional tiveram uma prevalência duas a três vezes maior de angina de peito, enfarte do miocárdio, doença arterial coronariana e hiperRuído tensão diastólica isolada. Após o ajuste para diversas covariáveis, a razão de chances (IC95\%) para a angina de peito, doença coronariana e hipertensão diastólica isolada foram 2,91 (1,35-6,26), 2,04 $(1,16-3,58)$ e 2,23 (1,21-4,12), respectivamente.

O estudo demonstrou que o IMC e o período de trabalho têm uma influência fundamental Hypertension in leather tanning workers working in Istanbul, Turkey ${ }^{25}$

Estudo 730 trabalhadores de transversal em Istambul, Turquia (2005)
Período de trabalho sobre o aumento do risco de hipertensão, o que leva a considerar a importância da exposição ocupacional.

Nos homens, a pressão arterial sistólica foi

481 trabalhadores de

Assessment of cardiometabolic risk among shift workers in Hungary ${ }^{14}$ turnos, muitos deles

Estudo transversal eram empregados na indústria leve ou nos serviços públicos
Trabalho em turnos maior nos trabalhadores noturnos em comparação aos trabalhadores diurnos. Nas mulheres, a taxa de prevalência de hipertensão na história médica foi maior nas trabalhadoras noturnas em comparação com as trabalhadoras diurnas.

The effects of urban bus driving on blood pressure and musculoskeletal problems: a quasi-experimental study ${ }^{26}$
88 motoristas de ônibus urbano no centro Coorte de Estocolmo, Suécia, por um período de 5 anos (2001-2006)
Número de horas dirigindo

90 policiais de

Study of occupational stress as a risk factor for various morbidities among policemen $^{19}$
Estudo transversal três estações policiais no distrito de Ahmednagar, Índia (2007)
O número médio de horas por semana dirigindo ônibus se apresentou como um preditor de maior PAD.
Sobrecarga de regra e responsabilidade foram moderadamente fatores de estresse entre todas as categorias analisadas. A pontuação de estresse foi significativamente alta entre policiais hipertensos. Assim, o estresse ocupacional é o fator de risco para o desenvolvimento de hipertensão. 
Tabela 1. Continuação.

\section{Título do artigo \\ Desenho do estudo \\ n do estudo População estudada \\ Tipo de \\ exposição ocupacional}

Cohort study for the effect of chronic noise exposure on blood pressure among male workers in Busan, Korea ${ }^{10}$
530 trabalhadores do sexo masculino

Coorte em uma indústria de produção de metal Busan, Coreia do Sul (1991-1999)
Resultados
Os valores médios para o PAS ao longo da duração deste estudo foram 3,8, 2,0, e 1,7 mmHg maior nos grupos NLC-IV, NLC-III, e NLC-II, respectivamente, em comparação com a do grupo NLC-I. Não houve diferença significativa na PAD entre os grupos. NCL-I: trabalhadores de escritório expostos a <60 dBA no trabalho; NLC-II: apoiadores técnicos ou inspetores que foram intermitentemente expostos a ruído e não estavam usando dispositivos de proteção auditiva; NCL-III: trabalhadores expostos a um nível de ruído abaixo de 85 dBA e que usaram um tipo dispositivo de proteção; NCL-IV: trabalhadores expostos a um nível de ruído de 85 dBA ou maior em média e usado tampão e protetor para as orelhas.

De acordo com resultados da Monitorização Ambulatorial da Pressão Arterial (MAPA), trabaIhar cansado algumas vezes, raramente/nunca diminui em 19,4 e 17,4\%, respectivamente, a
Estresse e privação de sono chance de apresentar pressão arterial alterada, em relação aos profissionais que referiram trabaIhar cansado frequentemente; sono diurno apareceu como um fator protetor diminuindo em 14,0\% a chance de apresentar hipertensão. em profissionais que atuam em serviços de atendimento pré-hospitalar ${ }^{20}$ transversal ambos da cidz São Paulo e SAMU Vale do Ribeira (SP) (2008-2009)
258 motoristas profissionais de trans-

Hipertensão arterial e obesidade em motoristas profissionais de transporte de cargas $^{27}$
Estudo transversal porte de cargas que trafegavam pela

Rodovia BR-116, no trecho Paulista-Regis Bittencourt
Uso de medicamento para inibir o sono
A hipertensão arterial se associou com: aumento do IMC, hiperglicemia; e hábito de ingerir medicamento para inibir o sono.
Comparadas com participantes com trabalho de baixa exigência, houve maior prevalência de hipertensão em trabalhos de alta exigência, 1.716 funcionários téc
Estresse no trabalho rial em mulheres no Estudo Pró-Saúde 22 e hipertensão arte-
Estudo transversal nico-administrati-vos de uma universidade no Estado do Rio de Janeiro (1999 e 2001)
Estresse passivos e ativos. Não houve associação estatisticamente significante entre diferentes categorias de estresse (alta exigência, ativo e passivo) e hipertensão arterial em nenhum dos modelos multivariados.
The relationship between working schedule patterns and the markers of the metabolic syndrome: comparison of shift workers ${ }^{15}$ workers with day

$\begin{array}{ccc}\text { Estudo } & \begin{array}{c}3.039 \text { motoristas do } \\ \text { oeste de Azerbaijão, }\end{array} & \begin{array}{c}\text { Trabalho em } \\ \text { transversal }\end{array}\end{array}$

O componente de hipertensão não foi significativamente relacionado com o trabalho em tur$\operatorname{nos}(p>0,05)$. 
Tabela 1. Continuação.

\begin{tabular}{|c|c|c|c|c|}
\hline Título do artigo & $\begin{array}{l}\text { Desenho } \\
\text { do estudo }\end{array}$ & $\begin{array}{c}\text { n do estudo } \\
\text { População estudada }\end{array}$ & $\begin{array}{c}\text { Tipo de } \\
\text { exposição } \\
\text { ocupacional }\end{array}$ & Resultados \\
\hline $\begin{array}{l}\text { Agravos à saúde, } \\
\text { hipertensão arterial } \\
\text { e predisposição ao } \\
\text { estresse em motoris- } \\
\text { tas de caminhão21 }\end{array}$ & $\begin{array}{l}\text { Estudo } \\
\text { transversal }\end{array}$ & $\begin{array}{l}258 \text { motoristas profis- } \\
\text { sionais de transporte } \\
\text { de cargas de rota } \\
\text { longa que trafegavam } \\
\text { pela Rodovia BR-116 no } \\
\text { trecho Paulista-Régis } \\
\quad \text { Bittencourt }\end{array}$ & Estresse & $\begin{array}{l}\text { Os resultados obtidos foram: 33\% eram portadores } \\
\text { de possíveis transtornos mentais comuns e houve } \\
\text { associação ( } p<0,05) \text { com referência de cansaço, } \\
\text { diminuição da concentração, considerar-se ner- } \\
\text { voso ou estressado, ter problemas pessoais ou no } \\
\text { trabalho e transportar carga com horário definido. } \\
\text { Não houve associação com hipertensão arterial. }\end{array}$ \\
\hline $\begin{array}{l}\text { The association of } \\
\text { shift work and hyper- } \\
\text { tension among male } \\
\text { factory workers in } \\
\text { Kota Bharu, Kelantan, } \\
\text { Malaysia16 }\end{array}$ & $\begin{array}{l}\text { Estudo } \\
\text { transversal }\end{array}$ & $\begin{array}{l}148 \text { trabalhadores } \\
\text { do sexo masculino } \\
\text { de uma fábrica em } \\
\text { Kota Bharu, Kelantan, } \\
\text { Malásia (2003-2004) }\end{array}$ & $\begin{array}{l}\text { Trabalho em } \\
\text { turnos }\end{array}$ & $\begin{array}{l}\text { A prevalência de hipertensão foi significativamente } \\
\text { maior entre os trabalhadores noturnos (22,4\%) } \\
\text { em comparação com os trabalhadores diurnos } \\
\text { (4,2\%), com valor p de 0,001. Turno de trabalho } \\
\text { foi significativamente associado com hipertensão } \\
\text { (odds ratio ajustada 9,1; IC95\% 1,4-56,7). }\end{array}$ \\
\hline
\end{tabular}
Malásia (2003-2004)

O risco na população de maior exposição foi 32\% maior do que a de base. 0 maior risco relativo foi de 1,5 em trabalhadores expostos por mais

Hypertension in noise -exposed sawmill workers: a cohort study ${ }^{12}$
10.872 trabalhado-

Coorte res de serrarias em Columbia, Canadá, 1991-1998
Ruído de 30 anos a 85 dBA. Tendências de exposição -resposta foram estatisticamente significativas. O risco de hipertensão foi positivamente associado com exposição a ruído acima de 85 dB.
The association between air pollution and blood pressure in traffic controllers in Santo André, São Paulo, Brazil24
Os aumentos da concentração de material particulado (PM1O) (33mg/m³) e de ozônio (49 mg/m³) foram associados com a elevação em todos os parâmetros de pressão arterial. A concentração de PM (10) foi associada com efeitos precoces principalmente sobre a PAS. No entanto, a concentração de ozônio foi mais fracamente associada com PAD.
Shift work is a risk factor for increased blood pressure in Japanese men: a 14-year historical cohort study ${ }^{17}$

\section{Estudo tráfego em Santo transversal (2008)}

Poluição do ar
3.963 trabalhadores diurnos e 2.748 de turnos alternados que receberam exames anuais de saúde entre 1991 e 2005 em uma empresa siderúrgica japonesa
Estudo em trabalhadores japoneses masculinos revelou que o trabalho por turnos alternados foi um fator de risco independente para um aumento da pressão arterial. Além disso, o efeito de trabalho em turnos sobre a pressão sanguínea foi mais pronunciado do que outros fatores bem conhecidos, tais como a idade e o IMC.

IC95\%: intervalo de confiança a 95\%; IMC: índice de massa corporal; PAS: pressão arterial sistólica; PAD: pressão arterial diastólica.

Após análise dos 23 artigos retidos, foi obtida a seguinte distribuição de acordo com o tipo de estudo: 18 transversais e 5 coortes. Quanto ao tipo de exposição ocupacional avaliada, trabalho em turnos, ruído e estresse corresponderam aos fatores ocupacionais mais abordados, presentes em 34,78, 30,43 e 26,08\% dos artigos, respectivamente. Outros fatores de riscos citados, em menor proporção, foram tempo de trabalho, solventes, poluição do ar e uso de medicamento para inibir o sono durante o trabalho. Ademais, cinco estudos analisaram dois ou mais desses fatores e sua relação com hipertensão $\operatorname{arterial}^{5,8,11,13,20}$.

Houve referência à associação positiva com HAS em 18 artigos, e negativa em um artigo ${ }^{27}$, de modo que em apenas quatro deles ${ }^{15,21-23}$ esses achados não foram observados. Para uma melhor análise dos resultados, esses estão descritos abaixo, de acordo com os principais fatores ocupacionais identificados. 


\section{TRABALHO EM TURNOS}

Foram retidos sete estudos ${ }^{5,11,13-17}$ sobre trabalho em turnos e HAS (dois coortes e cinco transversais), dos quais seis ${ }^{5,11,13,14,16,17}$ evidenciaram uma associação positiva. Além disso, em dois deles ${ }^{5,11}$ analisou-se a combinação com outros fatores ocupacionais como exposição a ruído, estresse no ambiente de trabalho e solventes.

Um dos artigos demonstrou um possível efeito aditivo entre ruído e trabalho em turnos para a ocorrência de HAS, estudando trabalhadores de uma indústria de manufatura de borracha. Foi realizada a comparação de grupos com diferentes exposições a trabalho em turnos (diurno e noturno) e a ruído (acima e abaixo de $85 \mathrm{~dB})^{5}$.

É válido ressaltar, ainda, 1 estudo de coorte histórico (14 anos) realizado no Japão, com 6.711 trabalhadores de fábrica de aço, no qual a alternância de turnos de trabalho constituiu fator independente para o incremento dos níveis tensionais ${ }^{17}$. Já outro trabalho (estudo transversal) analisou 3.039 motoristas iranianos e não demonstrou associação entre trabalhos em turnos e hipertensão arterial ${ }^{15}$.

Do total analisado, três artigos ${ }^{13,15-16}$ utilizaram inferência estatística com amostra randomizada em sua análise, e apenas um deles ${ }^{15}$ não evidenciou associação com HAS.

\section{RUÍDO}

Todos os oito estudos ${ }^{5-12}$ retidos sobre ruído e hipertensão demonstraram alguma associação positiva, inclusive aqueles que foram analisados em combinação com outros fatores de risco ocupacionais (trabalho em turnos e solventes) $)^{5,8,11}$. No entanto, os resultados encontrados são variáveis com relação aos seguintes aspectos: medida de frequências de ruído, nível em decibéis e alteração de pressão sistólica e/ou diastólica.

Um estudo realizado com 188 trabalhadores de uma fábrica de parafusos analisou a exposição a diferentes frequências e níveis de ruído e evidenciou associação positiva com hipertensão apenas nos expostos a valores maiores ou iguais a $70 \mathrm{~dB}$ na frequência de $4.000 \mathrm{~Hz}$ por 2 a 4 anos 7 . Já Lee et al. ${ }^{10}$ evidenciaram incremento de pressão arterial sistólica em 1,7, 2,0 e 3,8 $\mathrm{mmHg}$ respectivamente em trabalhadores expostos a ruído intermitente sem utilização de protetores auditivos, a níveis $<85$ e $>85 \mathrm{~dB}$ com uso de tais equipamentos, quando comparados ao grupo de base. Nesse mesmo estudo, não foi observada variação importante da pressão arterial diastólica ${ }^{10}$.
Sobre os métodos, apenas um dos trabalhos ${ }^{9}$ utilizou inferência estatística com amostra aleatória em sua análise. De todos os artigos examinados, apenas dois eram estudos de coorte ${ }^{10,12}$ e os demais eram estudos transversais.

\section{ESTRESSE}

Dos seis estudos avaliados sobre estresse no ambiente de trabalho ${ }^{13,18-22}$, quatro evidenciaram associação positiva com HAS $^{13,18-20}$. Um deles aponta como fator estressor o relacionamento na equipe de trabalho, que é fortemente associado à alteração da pressão sistólica e diastólica ${ }^{13}$.

Todos os artigos utilizaram questionários para avaliar a exposição ao estresse e em apenas dois deles ${ }^{21-22}$ a amostra não foi randomizada. Outros três direcionaram sua análise para a população feminina ${ }^{13,18,22}$. Quanto ao desenho, apenas um estudo foi de coorte ${ }^{13}$.

Ademais, entre os estudos retidos sobre o tema são apresentados diferentes conceitos de estresse, os quais foram abordados a partir dos seguintes aspectos: cansaço físico, demanda psicológica, controle no trabalho e organização.

As informações obtidas por questionário variaram de acordo com a definição de estresse utilizada e com os grupos profissionais analisados. Cavagioni L. et al. ${ }^{20}$, por exemplo, em artigo sobre hipertensão em profissionais de serviço pré-hospitalar, incluíram "trabalhar cansado" e "sono diurno durante o plantão" entre os itens avaliados. Este último, por sua vez, constituiu um fator protetor a partir da avaliação do período de sono por Monitorização Ambulatorial da Pressão Arterial $(\mathrm{MAPA})^{20}$.Já o estudo sobre estresse em policiais, realizado na Índia, analisou questões como condições de trabalho, prejuízo de relação afetiva, responsabilidade por terceiros e rentabilidade ${ }^{19}$.

\section{OUTROS}

Entre os demais artigos, cinco estudos ${ }^{8,11,24-26}$ também evidenciaram associação entre o fator de risco ocupacional analisado e a hipertensão. Trata-se de um total de seis artigos ${ }^{8,11,2427}$ sobre outros fatores de riscos ocupacionais, com a seguinte distribuição: tempo de trabalho (dois), solventes (um), poluição do ar (um) e uso de medicamentos para inibir o sono (um).

\section{DISCUSSÃo}

A HAS constitui uma doença crônica geralmente abordada a partir de fatores individuais (sedentarismo, obesidade, 
hereditariedade, entre outros) em detrimento daqueles relativos à coletividade (entre esses, os ocupacionais) como responsáveis pela sua patogênese. Entretanto, cada vez mais é discutida na literatura a possível relação entre riscos ocupacionais e o desenvolvimento da HAS. Nesse contexto, o presente estudo reuniu publicações de um período de cinco anos com a finalidade de agregar informações sobre essa temática.

A partir da análise dos resultados, observa-se que a maioria dos artigos incluídos nessa revisão evidenciou alguma associação entre fatores ocupacionais e hipertensão. Conforme já mencionado anteriormente, trabalho em turnos, ruído e estresse no ambiente de trabalho foram os riscos mais citados.

Diante dos estudos selecionados, evidenciou-se uma possível relação entre trabalho em turnos e hipertensão arterial, visto que seis dos sete estudos sobre o tema demonstraram essa associação. Em apenas um deles ${ }^{15} \mathrm{o}$ mesmo não foi observado, de modo a fortalecer a existência dessa associação. Ademais, nos dois $\operatorname{artigos~}^{5,11} \mathrm{em}$ que trabalho em turnos foi analisado em conjunto com outros fatores ocupacionais, houve associação positiva, o que pode sugerir uma contribuição do mesmo para o desenvolvimento de HAS também quando combinado a outros fatores de risco.

Vários estudos demonstram que o trabalho em turnos causa alteração do ritmo circadiano e, consequentemente, pode contribuir para o surgimento de doenças cardiometabólicas ${ }^{14,28-29}$, entre elas a hipertensão, em diferentes categorias profissionais. Outros fatores citados como relacionados a esse tipo de trabalho (distúrbios de sono e hábitos de vida menos saudáveis) contribuiriam na patogênese daquelas disfunções orgânicas ${ }^{14}$. Apesar da plausibilidade biológica das consequências do trabalho em turnos, o tema traz ainda controvérsias ${ }^{15}$.

Verificou-se que todos os sete estudos desta revisão sobre exposição a ruído e HAS evidenciaram alguma associação, apesar de grande heterogeneidade de resultados entre eles, relativa ao nível $(\mathrm{dB})$ e à frequência $(\mathrm{Hz})$ de ruído bem como ao período de exposição e ao efeito sobre as pressões arterial sistólica e/ou diastólica. Essa heterogeneidade de resultados dificulta a identificação de quais características da exposição a tal fator de risco estariam relacionadas a aumento de níveis pressóricos, já que os artigos apontaram associação em diferentes medidas de frequência e níveis de decibéis e, muitas vezes, relacionada à elevação de pressão sistólica ou diastólica isoladas. Ainda assim, pode-se afirmar que os achados fortalecem a relação entre ruído e hipertensão, visto que todos demonstraram o aumento dos níveis pressóricos com exposição ao ruído.

Sobre estresse no ambiente de trabalho, não foi possível estabelecer de forma conclusiva associação positiva com HAS a partir da análise dos seis artigos selecionados a respeito dessa temática. Observou-se que quatro de seis estudos apontaram relação entre estresse e hipertensão, de forma a não ser possível realizar tal inferência diante das evidências resumidas até aqui.

Entre os fatores que podem interferir nesses resultados está a utilização de diferentes conceitos de estresse - fadiga, demanda psicológica e de controle, biológico (sintomas físicos e psíquicos) - entre os estudos, de forma a não haver uniformidade entre os aspectos avaliados por meio de questionário. Ademais, cada artigo avaliou categorias profissionais específicas e, portanto, algumas das informações obtidas foram direcionadas para o grupo analisado, impossibilitando inferências amplas sobre a relação entre estresse e hipertensão.

Os demais fatores ocupacionais somaram seis artigos, com a seguinte distribuição: tempo de trabalho (dois), solventes (dois), poluição do ar (um) e uso de medicamentos para inibir o sono (um). O pequeno número desses estudos impossibilitou a análise da relação com o aumento dos níveis pressóricos.

Ademais, a maioria dos artigos utilizados nesta revisão trata de estudos transversais, que apresentam algumas limitações válidas de ressalva. Esse tipo de desenho não permite estabelecer relação de causalidade entre a exposição e o desfecho. Também o efeito trabalhador sadio pode estar presente, já que os trabalhadores incluídos na população estudada poderiam ser aqueles ativos no momento da coleta dos dados.

Outro ponto importante a ser ressaltado é que o uso de inferência estatística não se aplica a pesquisa que não utiliza amostragem aleatória para obtenção da população de estudo. Apesar disso, em vários estudos ${ }^{7-8,17,22,23,27}$ os autores, de forma equivocada, recorrem aos testes estatísticos. A conveniência de uma amostra impossibilita fazer uso da inferência estatística dos resultados, já que ela não foi obtida atendendo aos pressupostos da estatística inferencial. Da mesma forma, em caso de estudos que abordam 
o universo de indivíduos (um censo) ( $-6,10,12,25$ não há porque se fazer testes estatísticos, tendo em vista que os resultados foram obtidos para todos os indivíduos da população e, portanto, não foi retirada uma porção de um universo para o qual pretende-se inferir estatisticamente os resultados obtidos na amostra.

A realização de teste estatístico só pode ter sentido para inferir os resultados para a população-alvo, a partir de amostra aleatória. Assim, o que se pode fazer em estudos com amostra por conveniência é a inferência não estatística - os autores têm elementos que sugerem que a amostra por conveniência utilizada "representa" ou "se aproxima" do universo da população? Mas isso não é inferência estatística. Embora esse aspecto usar inferência estatística para resultados de amostras não aleatórias - revele uma inadequação metodológica, especificamente de análise dos dados, presente em muitos estudos da revisão, optou-se por mantê-los, tendo em vista o predomínio dessa inadequação na literatura epidemiológica ${ }^{30}$.

\section{CONCLUSÃO}

Com base nos achados deste estudo, conclui-se que, dentre os fatores ocupacionais em questão, o ruído se destacou como associado à hipertensão arterial assim como, em menor proporção, o trabalho em turnos. Quanto aos demais, não foi possível concluir sobre a existência ou não dessa relação. Assim, são necessários mais estudos sobre o tema - HAS e fatores ocupacionais - para ampliar a compreensão dessa problemática.

Diante da elevada prevalência de HAS na população brasileira, o controle e o tratamento dessa patologia como fator de risco modificável seriam de impacto na redução da morbimortalidade por doenças cardiovasculares. Nesse contexto, a saúde do trabalhador se insere no cenário da saúde pública não apenas ante à significativa morbimortalidade e aos custos para a saúde oriundos de doenças cardiovasculares, mas também diante das possibilidades de ação preventiva e de controle de riscos resultantes da exposição ocupacional a possíveis fatores predisponentes.

\section{REFERÊNCIAS}

1. Brasil. Ministério da Saúde. Secretaria de Vigilância em Saúde. [Internet] Vigitel Brasil 2011: Vigilância de fatores de risco e proteção para doenças crônicas por inquérito telefônico [acesso em 2013 maio 12]. Brasília: Ministério da Saúde; 2012. Disponível em: <http:// portalsaude.saude.gov.br/portalsaude/arquivos/pdf/2012/Ago/22/ vigitel_2011_final_0812.pdf>

2. Cordeiro R, Fischer FM, Filho EC, Filho DJ. [Internet] Ocupação e hipertensão [acessoem 2013maio 12]. Rev.SaúdePública. 1993;27(5):380-7. Disponível em: <http://www.scielo.br/pdf/rsp/v27n5/10.pdf>

3. Brasil. Ministério da Saúde. [Internet] Plano Nacional de Saúde (PNS) 2012-2015 [acesso em 2013 maio 12]. Brasília, 2011. Disponível em: <http://conselho.saude.gov.br/biblioteca/Relatorios/plano_ nacional_saude_2012_2015.pdf>

4. Sociedade Brasileira de Cardiologia / Sociedade Brasileira de Hipertensão / Sociedade Brasileira de Nefrologia. [Internet] VI Diretrizes Brasileiras de Hipertensão [acesso em 2013 maio 12]. Arq Bras Cardiol. 2010;5(1Suppl.1):1-51. Disponível em:<http://publicacoes. cardiol.br/consenso/2010/Diretriz_hipertensao_associados.asp>

5. Attarchi M, Dehghan F, Safakhah F, Nojomi M, Mohammadi S. Effect of exposure to occupational noise and shift working on blood pressure in rubber manufacturing company workers. Ind Health. 2012;50(3):205-13.

6. Cheng T, Liu C, Huang K, Chen R, Lai J, Bao B. High frequency hearing loss, occupational noise exposure and hypertension: A cross sectional study in male workers. Environmental Health. 2011;10:35-42.
7. Chang T, Liu C, Young L, Wang V, Jian S, Bao B. Noise frequency components and the prevalence of hypertension in workers. Sci Total Environ. 2012;416:89-96.

8. Chang T, Wang V, Hwang B, Yen H, Lai J, Liu C et al. Effects of co-exposure to noise and mixture of organic solvents on blood pressure. J Occup Health. 2009;51:332-9.

9. Gan W, Davies H, Demers P, Brauer M. Exposure to Occupational Noise and Cardiovascular Disease in the United States: the National Health and Nutrition Examination Survey 1999-2004. Occup Environ Med. 2011;68(3):183-90.

10. Lee J, Kang W, Yaang S, Choi N, Lee CR. Cohort study for the effect of chronic noise exposure on blood pressure among male workers in Busan, Korea. Am J Ind Med. 2009;52(6):509-17.

11. Sancini A, Tomei G, Vitarelli A, Caciari T, Samperi I, Pacchiarotti A et al. Cardiovascular risk in rotogravure industry. J Occup Environ Med. 2012 May;54(5):551-7.

12. Sbihi H, Davies H, Demers P. Hypertension in noise-exposed sawmill workers: a cohort study. Occup Environ Med. 2008;65(9):643-6.

13. De Gaudemaris R, Levant A, Ehlinger V, Hérin F, Lepage B, Soulat $J$, et al. Blood pressure and working conditions in hospital nurses and nursing assistants. The ORSOSA study. Arch Cardiovasc Dis. 2011;104(2):97-103.

14. Jermendy G, Nádas J, Hegyi I, Vasas I, Hidvégi T. Assessment of cardiometabolic risk among shift workers in Hungary. Health Qual Life Outcomes. 2012;10(1):1-6. 
15. Mohebbi I, Shateri K, Seyedmohammadzad M. The relationship between working schedule patterns and the markers of the metabolic syndrome: Comparison of shift workers with day workers. Int J Occup Med Environ Health. 2012;25(4):383-91.

16. Nazri S, Tengku M, Winn T. The association of shift work and hypertension among male factory workers in Kota Bharu, Kelantan, Malaysia. Southeast Asian J Trop Med Public Health. 2008;39:176-83.

17. Suwazono Y, Dochi M, Sakata K, Okubo Y, Oishi M, Tanaka K et al. Shift work is a risk factor for increased blood pressure in japanese men a 14-year historical cohort study. Hypertension. 2008; 52(3):581-6.

18. Bojar I, Humeniuk E, Owoc A, Wierzba W, Wojtyla A. Exposing women to workplace stress factors as a risk factor for developing arterial hypertension. Ann Agric Environ Med. 2011 Jun; 18(1):175-82.

19. Kamble S, Phalke D. Study of occupational stress as a risk factor for various morbidities among policemen. J Indian Med Assoc. 2011;109(4):238.

20. Cavagioni L, Pierin A. Hipertensão arterial em profissionais que atuam em serviços de atendimento pré-hospitalar. Texto contexto enferm. 2011;20(3):235.

21. Cavagioni L, Pierin A, Batista K, Bianch E, Costa A. Agravos à saúde, hipertensão arterial e predisposição ao estresse em motoristas de caminhão. Rev Esc Enferm. 2009;43: 1267-71.

22. Faerstein E, Werneck G, Lopes C. Estresse no trabalho e hipertensão arterial em mulheres no Estudo Pró-Saúde. Rev Saúde Pública. 2009;43(5):893-6.
23. Wenzel D, de Souza J, de Souza S. Prevalência de hipertensão arterial em militares jovens e fatores associados. Rev Saúde Pública. 2009;43(5):789-95.

24. Chiarelli P, Pereira L, Saldiva P, Filho C, Garcia M, Braga A et al. The association between air pollution and blood pressure in traffic controllers in Santo André, São Paulo, Brazil. Environmental Research. 2011;111(5):650-5.

25. Ince $\mathrm{N}$, Işsever $\mathrm{H}$, Ince $\mathrm{H}$, Özyildirim B, Isik E, Hapcioglu B et al. Hypertension in leather tanning workers working in Istanbul, Turkey. Singapore Med J. 2008:49(11):874-8.

26. Johansson G, Evans G, Cederström C, Rydstedt L, Fuller-Rowell T, Ong A. The effects of urban bus driving on blood pressure and musculoskeletal problems: a quasi-experimental study. Psychosom Med.2012;74(1):89-92.

27. Cavagioni L, Pierin A. Hipertensão arterial e obesidade em motoristas profissionais de transporte de cargas. Acta Paul Enferm. 2010:23(4):455-60.

28. Sookoian S, Gemma C, Fernández Gianotti T, Burgueño A, Alvarez A, González C et al. Effects of rotating shift work on biomarkers of metabolic syndrome and inflammation. J Intern Med. 2007;261:285-92.

29. Boggild H, Knutsson A. Shift work, risk factors and cardiovascular disease. Scand J Work Environ Health. 1999;25:85-99.

30. Conceição MJ. Leitura crítica dos dados estatísticos em trabalhos científicos. Rev Bras Anestesiol. 2008;58:260-6.

Endereço para correspondência: Roberta Coimbra Velez de Andrade Faculdade de Medicina da Universidade Federal da Bahia (UFBA) - Largo Terreiro de Jesus s/n, Pelourinho - CEP: 40020-210 - Salvador (BA), Brasil -

E-mail: robertacvelez@gmail.com 